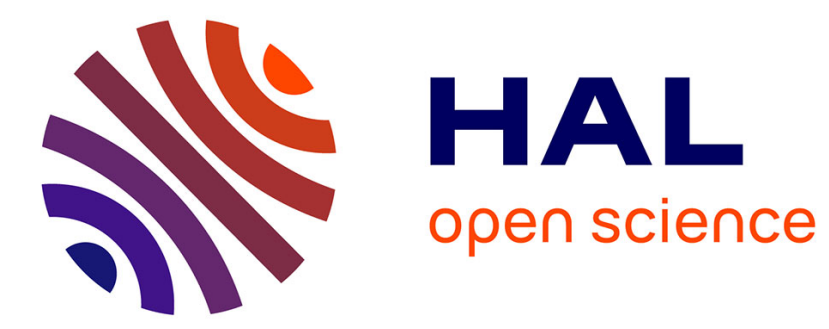

\title{
COURANTS NEUTRES ET CHARME DANS LES INTERACTIONS DE NEUTRINOS
}

B. Degrange

\section{To cite this version:}

B. Degrange. COURANTS NEUTRES ET CHARME DANS LES INTERACTIONS DE NEUTRINOS. Congress Of The French Physical Society, 1977, Poitiers, France. pp.C3-79-C3-91, 10.1051/jphyscol:1978311 . jpa-00217415

\section{HAL Id: jpa-00217415 https://hal.science/jpa-00217415}

Submitted on 1 Jan 1978

HAL is a multi-disciplinary open access archive for the deposit and dissemination of scientific research documents, whether they are published or not. The documents may come from teaching and research institutions in France or abroad, or from public or private research centers.
L'archive ouverte pluridisciplinaire HAL, est destinée au dépôt et à la diffusion de documents scientifiques de niveau recherche, publiés ou non, émanant des établissements d'enseignement et de recherche français ou étrangers, des laboratoires publics ou privés. 


\title{
COURANTS NEUTRES ET CHARME DANS LES INTERACTIONS DE NEUTRINOS
}

\author{
B. DEGRANGE
}

\author{
LPNHE, École Polytechnique, 91128 Palaiseau, France
}

\begin{abstract}
Résumé. - Depuis quatre ans, la Physique du Neutrino a connu deux développements importants : l'étude des processus dus aux courants neutres faibles et l'étude de la production de particules charmées par des neutrinos. Pour illustrer les progrès expérimentaux accomplis dans ces domaines jusqu'en juin 1977, nous présentons dans cet article quelques résultats choisis parmi les plus marquants.
\end{abstract}

\begin{abstract}
During the last four years, Neutrino Physics has been developed into the main following directions : the study of neutral-current-induced processes, and the study of charmed particle production by neutrinos. The experimental progresses obtained in those fields up to June 1977 are illustrated by some outstanding results.
\end{abstract}

1. Introduction. - Les résultats expérimentaux obtenus depuis quatre ans en Physique du Neutrino ont confirmé une succession remarquable de prédictions théoriques. La première de ces prédictions était l'existence de courants neutres, élément indispensable des théories renormalisables de l'interaction faible, tant qu'on ne désire pas introduire de nouveau lepton [1]. C'est l'étude des réactions de neutrinos, réalisée au CERN dans la chambre à bulles Gargamelle qui a prouvé en 1973 l'existence de courants neutres [2], en mettant en évidence des réactions de la forme: $\nu_{\mu}\left(\mathrm{ou} \bar{\nu}_{\mu}\right)+\mathrm{N} \rightarrow \nu_{\mu}$ (ou $\left.\bar{\nu}_{\mu}\right)+$ hadrons. Toutefois, l'étude de la désintégration des particules étranges avait montré que les courants neutres changeant l'étrangeté devaient être exclus au premier ordre de l'interaction faible [3]. Dès 1970, Glashow, Iliopoulos et Maiani avaient proposé un modèle simple dans lequel les courants neutres conservaient l'étrangeté [4] ; ce résultat était obtenu au prix de l'introduction d'un nouveau « quark » $\mathbf{p}^{\prime}$, dit « charmé », qui venait s'ajouter aux trois quarks $\mathrm{p}, \mathrm{n}, \lambda$ expliquant les hadrons déjà connus. On obtenait donc une seconde série de prédictions concernant l'existence et les propriétés d'une nouvelle farnille de particules charmées, susceptible en particulier d'être produites par des neutrinos dans des processus de type courant chargé. En 1974, la découverte d'interactions de neutrinos donnant deux leptons de signes opposés dans l'état final [5] constituait le premier indice sérieux d'une telle production ; cette interprétation était considérablement renforcée en 1976 par l'observation de particules étranges dans les événements à deux leptons [6]. Enfin pendant l'été 1976, les expériences auprès des anneaux de collisions $\mathrm{e}^{+} \mathrm{e}^{-}$à Stanford [7] et une expérience de photoproduction à Fermi Lab. [8] fournissaient les masses des premières particules charmées.
La découverte des courants neutres comme celle du charme ouvrent des voies nouvelles de recherche en Physique du Neutrino ; c'est aux résultats acquis aujourd'hui dans chacune d'elles que nous consacrerons les deux parties qui suivent. Par ailleurs, des expériences ont récemment signalé la présence de phénomènes difficilement interprétables dans le cadre théorique précédent, en particulier la production de deux leptons de même signe, voire de trois leptons. Tous ces résultats sont aujourd'hui encore controversés ; nous consacrerons une dernière partie à la discussion de la situation expérimentale actuelle dans ce domaine.

2. Courants neutres dans les réactions de neutrinos. - Les réactions de neutrino induites par les courants neutres sont celles dans lesquelles le neutrino incident redonne un neutrino dans l'état final, au lieu de se transformer en lepton chargé (muon ou électron) comme dans les processus dus aux courants chargés.

Nous présenterons d'abord les résultats concernant les réactions sur des électrons $\left(\left(\nu_{\mu}\right.\right.$ ou $\left.\bar{\nu}_{\mu}\right)+\mathrm{e}^{-}$et $\left(\nu_{\mathrm{e}}\right.$ ou $\left.\left.\bar{\nu}_{\mathrm{e}}\right)+\mathrm{e}^{-}\right)$; en effet, malgré la faiblesse de leurs sections efficaces, ces processus ont l'avantage de ne mettre en jeu que des leptons et constituent par suite les tests les plus directs des divers modèles théoriques. Nous considérerons ensuite les réactions de neutrinos sur des nucléons, qui, dans le cadre des connaissances actuelles sur la structure des hadrons, permettent d'acquérir d'importantes informations sur les propriétés d'espace-temps et sur l'isospin des courants neutres. Nous présenterons enfin un test récent de la conservation de l'étrangeté par les courants neutres dans les réactions de neutrino.

Tous ces résultats sont interprétés en faisant l'hypothèse que les courants neutres comportent uniquement une partie vectorielle (V) et une partie 
axiale (A), ainsi que le prédisent la plupart des modèles. Il n'est pas possible en effet, dans le cadre des données actuelles, de faire une analyse entièrement phénoménologique [9].

2.1 COURANTS NEUTRES DANS LES RÉACTIONS ENTRE LEPTONS. - Les réactions purement leptoniques aujourd'hui étudiées sont les suivantes :

a) $\nu_{\mu}\left(\right.$ ou $\left.\bar{\nu}_{\mu}\right)+\mathrm{e}^{-} \rightarrow \nu_{\mu}\left(\right.$ ou $\left.\bar{\nu}_{\mu}\right)+\mathrm{e}^{-}$, processus uniquement dû au courant neutre, décrit par le graphe de la figure 1(a) et par le lagrangien effectif suivant $[10]$ :

$$
L_{\text {eff }}=-\frac{G}{\sqrt{2}} \bar{\nu}_{\mu} \gamma_{\lambda}\left(1+\gamma_{s}\right) \nu_{\mu} \cdot \bar{e} \gamma_{\lambda}\left(g_{V}+g_{\mathrm{A}} \gamma_{s}\right) e
$$

dans lequel $G$ est la constante de Fermi, les $\gamma$ sont les matrices de Dirac, et $g_{\mathrm{V}}$ et $g_{\mathrm{A}}$ les constantes de couplage vectorielle et axiale du courant neutre de l'électron.

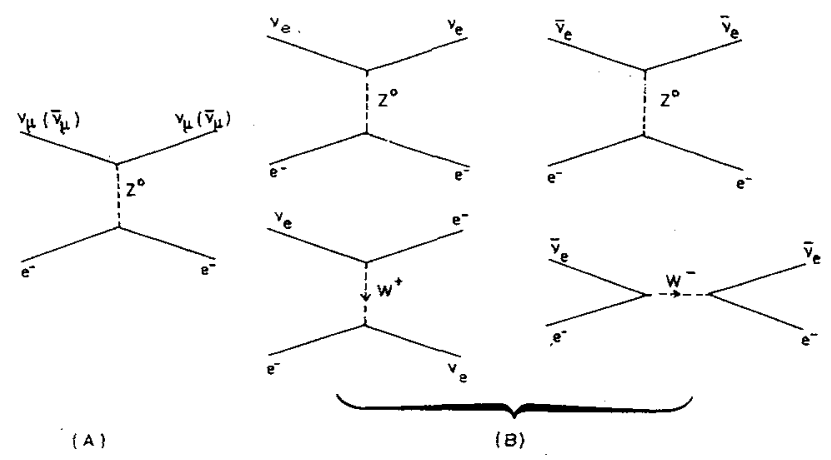

FIG. 1. - Graphes contribuant aux réactions suivantes : (a) $\nu_{\mu}$ (ou $\left.\bar{\nu}_{\mu}\right)+\mathrm{e}^{-} \rightarrow \nu_{\mu}\left(\right.$ ou $\left.\bar{\nu}_{\mu}\right)+\mathrm{e}^{-}$, processus purement « courant neutre ». (b) $\nu_{\mathrm{e}}\left(\mathrm{ou} \bar{\nu}_{\mathrm{e}}\right)+\mathrm{e}^{-} \rightarrow \nu_{\mathrm{e}}\left(\mathrm{ou} \bar{\nu}_{\mathrm{e}}\right)+\mathrm{e}^{-}$, processus dû partiellement au courant neutre et partiellement au courant chargé.

[Graphs contributing to the following reactions :

$$
\text { (a) } \left.\left.\nu_{\mu} \text { (or } \bar{\nu}_{\mu}\right)+\mathrm{e}^{-} \rightarrow \nu_{\mu} \text { (or } \bar{\nu}_{\mu}\right)+\mathrm{e}^{-} \text {, }
$$

a purely neutral current-induced-process.

(b) $\nu_{\mathrm{e}}\left(\right.$ or $\left.\bar{\nu}_{\mathrm{e}}\right)+\mathrm{e}^{-} \rightarrow \nu_{\mathrm{e}}\left(\right.$ or $\left.\bar{\nu}_{\mathrm{e}}\right)+\mathrm{e}^{-}$

a process due partly to neutral currents, partly to charged currents.]

b) $\nu_{\mathrm{e}}\left(\right.$ ou $\left.\quad \bar{\nu}_{\mathrm{e}}\right)+\mathrm{e}^{-} \rightarrow \nu_{\mathrm{e}}\left(\right.$ ou $\left.\quad \bar{\nu}_{\mathrm{e}}\right)+\mathrm{e}^{-}$, réaction à laquelle contribuent à la fois le processus courant neutre et le processus courant chargé décrits par les graphes de la figure 1(b). Le lagrangien effectif après remaniement des termes par transformation de Fierz peut s'écrire [10]:

$$
\begin{aligned}
L_{\text {eff }}=-\frac{G}{\sqrt{2}} \bar{\nu}_{e} \gamma_{\mu}\left(1+\gamma_{s}\right) \nu_{\mathrm{e}} \cdot \bar{e} \gamma_{\mu} & {\left[\left(1+g_{\mathrm{v}}\right)\right.} \\
+ & \left.\left(1+g_{\mathrm{A}}\right) \gamma_{s}\right] e
\end{aligned}
$$

expression qui ne diffère de (1) que par le remplacement de $g_{\mathrm{V}}$ en $1+g_{\mathrm{V}}$ et de $g_{\mathrm{A}}$ en $1+g_{\mathrm{A}}$.

Les sections efficaces $\sigma$ ainsi prédites sont proportionnelles à l'énergie $E$ du neutrino incident et s'expriment en fonction de $g_{\mathrm{V}}$ et $g_{\mathrm{A}}$ selon [10]:

$$
\begin{aligned}
\sigma\left(\nu_{\mu}+\mathrm{e}^{-}\right) & =\frac{G^{2} m_{\mathrm{e}} E}{2 \pi} \times \\
\times & {\left[\left(g_{\mathrm{V}}+g_{\mathrm{A}}\right)^{2}+\frac{1}{3}\left(g_{\mathrm{V}}-g_{\mathrm{A}}\right)^{2}-\frac{m_{\mathrm{e}}}{2 E}\left(g_{\mathrm{V}}^{2}-g_{\mathrm{A}}^{2}\right)\right] }
\end{aligned}
$$

$$
\begin{aligned}
\sigma\left(\bar{\nu}_{\mu}+\mathrm{e}^{-}\right) & =\frac{G^{2} m_{\mathrm{e}} E}{2 \pi} \times \\
\times & {\left[\left(g_{\mathrm{V}}-g_{\mathrm{A}}\right)^{2}+\frac{1}{3}\left(g_{\mathrm{V}}+g_{\mathrm{A}}\right)^{2}-\frac{m_{\mathrm{e}}}{2 E}\left(g_{\mathrm{V}}^{2}-g_{\mathrm{A}}^{2}\right)\right] }
\end{aligned}
$$

Le dernier terme proportionnel à la masse $m_{\mathrm{e}}$ de l'électron est négligeable pour $E \sim 1 \mathrm{GeV}$. Si l'on connaît le spectre en énergie des neutrinos, le nombre d'événements observés donne une contrainte qui se traduit dans le plan $\left(g_{\mathrm{V}}, g_{\mathrm{A}}\right)$ par une ellipse (dont les axes sont les bissectrices pour $E \gg m_{e}$ ). Pour les réactions avec des neutrinos de type électron, la conclusion est analogue, mais l'ellipse est centrée sur le point de coordonnées $(-1,-1)$, comme on le voit en comparant les formules (1) et (2). Les rapports $\sigma / E$ prédits sont de l'ordre de $10^{-42} \mathrm{~cm}^{2} / \mathrm{GeV}$, ce qui explique la faiblesse des statistiques disponibles aujourd'hui pour ces événements purement leptoniques.

A l'heure actuelle, deux expériences dans le faisceau neutrino du PS du CERN ont fourni des résultats sur les réactions $\left(\nu_{\mu}\right.$ ou $\left.\bar{\nu}_{\mu}+\mathrm{e}^{-}\right)$:

2.1.1 L'expérience réalisée dans la chambre à bulles Gargamelle [11] permet une identification claire des événements, car le vertex de l'interaction est bien visible et la distinction entre électron, positron et $\gamma$ est aisée aux énergies intéressantes. Ceci limite considérablement la contribution d'événements de bruit de fond tels que : $\nu_{c}+n \rightarrow e^{-}+p$ (détection du proton au vertex, sauf à très basse énergie) ou $\nu_{\mu}+\mathrm{N} \rightarrow \nu_{\mu}+\mathrm{N}+\pi^{0}\left(\pi^{0} \rightarrow \gamma \gamma\right)$ (bonne détection des $\gamma$ et bonne distinction électron $-\gamma$ ). En revanche la statistique est très réduite; (3 événements $\bar{\nu}_{\mu}+\mathrm{e}^{-}, 1$ événement $\nu_{\mu}+\mathrm{e}^{-}$).

2.1.2 L'expérience de la collaboration Aix-laChapelle-Padoue [12], réalisée avec des chambres à étincelles et sans champ magnétique dispose d'une statistique un peu plus grande, mais est soumise à une contamination plus forte en raison de la moins bonne visibilité du vertex et de l'impossibilité de distinguer les électrons des positrons ou des $\gamma$. Des résultats très récents de Juin 1977 [13] portent sur 9 événements $\bar{\nu}_{\mu}+\mathrm{e}^{-}$et 11 événements $\nu_{\mu}+\mathrm{e}^{-}$avec environ $30 \%$ de bruit de fond. Les résultats de ces deux expériences sont indiqués dans le tableau I. Sur la figure 2 , on a reporté dans le plan $\left(g_{\mathrm{V}}, g_{\mathrm{A}}\right)$ les intervalles de confiance à $90 \%$ donnés par l'expérience de Gargamelle.

Sur la réaction $\bar{\nu}_{\mathrm{e}}+\mathrm{e}^{-} \rightarrow \bar{\nu}_{\mathrm{e}}+\mathrm{e}^{-}$on dispose des résultats de l'expérience de Reines et al. [14] auprès du réacteur de Savannah River. Ceux-ci délimitent un autre intervalle de confiance dans le plan $\left(g_{\mathrm{V}}\right.$, $g_{\mathrm{A}}$ ) ; on a reporté sur la figure 2 l'intervalle à $68 \%$ de confiance donné par les auteurs [14].

Le modèle de Salam et Weinberg [15] prédit

$$
g_{\mathrm{A}}=-\frac{1}{2}, \quad \text { et } \quad g_{\mathrm{v}}=-\frac{1}{2}+2 \sin ^{2} \theta_{\mathrm{w}},
$$




\section{TABLEAU I}

Principaux résultats des expériences sur les processus purement leptoniques : $\nu_{\mu}\left(\bar{\nu}_{\mu}\right)+\mathrm{e}^{-}$et $\overline{\nu_{\mathrm{e}}}+\mathrm{e}^{-}$.

[Experimental results on purely leptonic processes : $\nu_{\mu}\left(\bar{\nu}_{\mu}\right)+\mathrm{e}^{-}$and $\left.\overline{\nu_{\mathrm{e}}}+\mathrm{e}^{-}.\right]$

\begin{tabular}{lc}
\multicolumn{1}{c}{ Expérience } & Statistique \\
Gargamelle & - \\
$\left(\nu_{\mu}, \bar{\nu}_{\mu} /\right.$ CERN $)$ & $\nu_{\mu}: 1$ \\
& $\bar{\nu}_{\mu}: 3$ \\
Aix-la-Chapelle & \\
Padoue & $\nu_{\mu}: 11$ \\
$\left(\nu_{\mu}, \bar{\nu}_{\mu} /\right.$ CERN $)$ & $\bar{\nu}_{\mu}: 9$
\end{tabular}

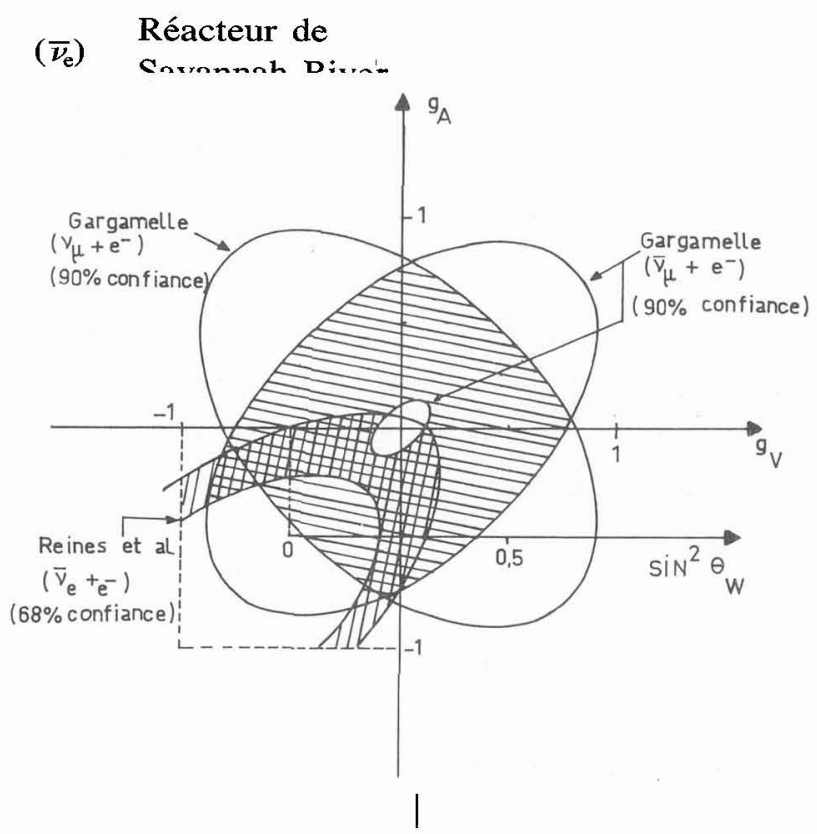

FrG. 2. - Limites expérimentales sur les constantes de couplage axiale $\left(g_{\mathrm{A}}\right)$ et vectorielle $\left(g_{\mathrm{V}}\right)$ du courant neutre de l'électron.

[Experimental limits on axial $\left(g_{\mathrm{A}}\right)$ and vector $\left(g_{\mathrm{V}}\right)$ coupling constants in the electron neutral current.]

où $\theta_{\mathrm{w}}$ est l'angle de Weinberg; on vérifie sur le tableau I que les données actuelles favorisent

$$
\sin ^{2} \theta_{\mathrm{w}} \sim 0,3 \text {. }
$$

2.2 COURANTS NeUtres ET STRUCTURE HADRONIQUE. - L'interprétation des réactions neutrinonucléon en courant neutre ne peut se faire qu'à partir de la connaissance de la structure des nucléons. Celle-ci a fait l'objet de nombreuses études [16], tant en électroproduction qu'en neutrino-production due aux courants chargés, qui ont accrédité l'image d'un nucléon constitué de 3 quarks de valence (ppn pour le proton, nnp pour le neutron) et d'une faible contribution de paires quark-antiquark (ou mer).

2.2.1 Contribution des courants gauche et droit. - Le tableau II rappelle les comportements du courant électromagnétique ( $\mathrm{V}$ pur) et du courant faible chargé ( $V-A$ pur) par rapport à la structure

$$
\begin{gathered}
\sigma / E\left(\text { en } 10^{-42} \mathrm{~cm}^{2} / \mathrm{GeV}\right) \\
- \\
v_{\mu}:<3,4 \\
\bar{\nu}_{\mu}: 1_{-0,9}^{+2,1}
\end{gathered}
$$

( $90 \%$ de confiance)

$$
\begin{aligned}
& \nu_{\mu}: 1,5 \pm 0,9 \\
& \bar{\nu}_{\mu}: 3,0 \pm 1,3
\end{aligned}
$$

$\sin ^{2} \theta_{\mathrm{w}}$

$<0,4$

(90\% de confiance)

$$
\begin{aligned}
& 0,37 \pm 0,13 \\
& 0,38 \pm 0,20
\end{aligned}
$$

$$
\bar{\nu}_{\mathrm{e}}: 5,8 \pm 1,4
$$

$0,29 \pm 0,05$

du nucléon. Ce dernier courant permet de distinguer quarks et antiquarks, comme on le constate sur les distributions $\mathrm{d} \sigma / \mathrm{d} y$ de la variable $y$ de Bjorken, rapport de l'énergie des hadrons produits à l'énergie incidente, et sur les valeurs des sections efficaces inclusives. Les courants neutres diffèrent des courants chargés en ce qu'ils comportent a priori une partie V-A (ou gauche) et une partie V + A (ou droite). L'une des questions essentielles est de savoir si les contributions gauche et droite sont ou non égales, leur égalité signifiant la conservation de la parité (V pur ou $A$ pur), prédite d'ailleurs par certains modèles [17]. L'examen du tableau II montre que dans ce dernier cas, quelle que soit la proportion d'antiquarks dans le nucléon, les sections efficaces inclusives $\sigma_{\mathbb{N}}=\sigma\left(\nu_{\mu}+\mathrm{N} \rightarrow \nu_{\mu}+\cdots\right)$ et $\bar{\sigma}_{\mathrm{N}}=\sigma\left(\bar{\nu}_{\mu}+\mathrm{N} \rightarrow \bar{\nu}_{\mu}+\cdots\right)$ doivent être égales. D'où l'intérêt de la mesure du rapport $R_{\mathrm{N}}=\sigma_{\mathrm{N}} / \bar{\sigma}_{\mathrm{N}}$.

Les résultats expérimentaux sont résumés dans le tableau III ; pour être moins sensible aux divers critères d'acceptance utilisés, on préfère généralement mesurer les rapports de chaque section efficace inclusive en courant neutre ( $\sigma_{\mathrm{N}}$ et $\bar{\sigma}_{\mathrm{N}}$ ) à la section efficace correspondante en courant chargé, $\left(\sigma_{\mathrm{c}}=\sigma\left(\nu_{\mu}+\mathrm{N} \rightarrow \mu-\cdots\right) \quad\right.$ et $\quad \bar{\sigma}_{\mathrm{c}}=\sigma\left(\bar{\nu}_{\mu}+\mathrm{N} \rightarrow\right.$ $\left.\left.\mu^{+}+\cdots\right)\right)$. Tous les rapports donnés dans le tableau III sont en excellent accord ; ceci est remarquable dans la mesure où l'expérience réalisée au PS du CERN dans Gargamelle [18] avec des neutrinos de 1 à $12 \mathrm{GeV}$ donne des rapports compatibles avec les deux expériences à haute énergie de Fermi Lab., l'une dans un faisceau à bande large [19], l'autre dans un faisceau dichromatique [20], de même qu'avec l'expérience réalisée au SPS du CERN avec le détecteur de $900 \mathrm{t}$ de la Collaboration CERN-Dortmund-Heidelberg-Saclay [21] (CDHS) en faisceau dichromatique. Les rapports $\sigma_{N} / \sigma_{c}$ et $\bar{\sigma}_{\mathrm{N}} / \bar{\sigma}_{\mathrm{c}}$ restent donc constants de quelques $\mathrm{GeV}$ à près de $200 \mathrm{GeV}$, conformément à ce qu'on attend de l'invariance d'échelle. Tous ces résultats concordent pour établir la violation de la parité dans les réactions courant neutre; on peut le voir sur la 


\section{TABLEAU II}

Comportements des courants électromagnétique et faibles par rapport aux constituants du nucléon: distributions en $y$ et sections efficaces inclusives $(\nu / \bar{\nu})$.

[Characteristics of the electromagnetic and weak currents with respect to the constituents of the nucleon : $y$ distributions and $(\nu / \bar{\nu})$ inclusive cross-sections.]

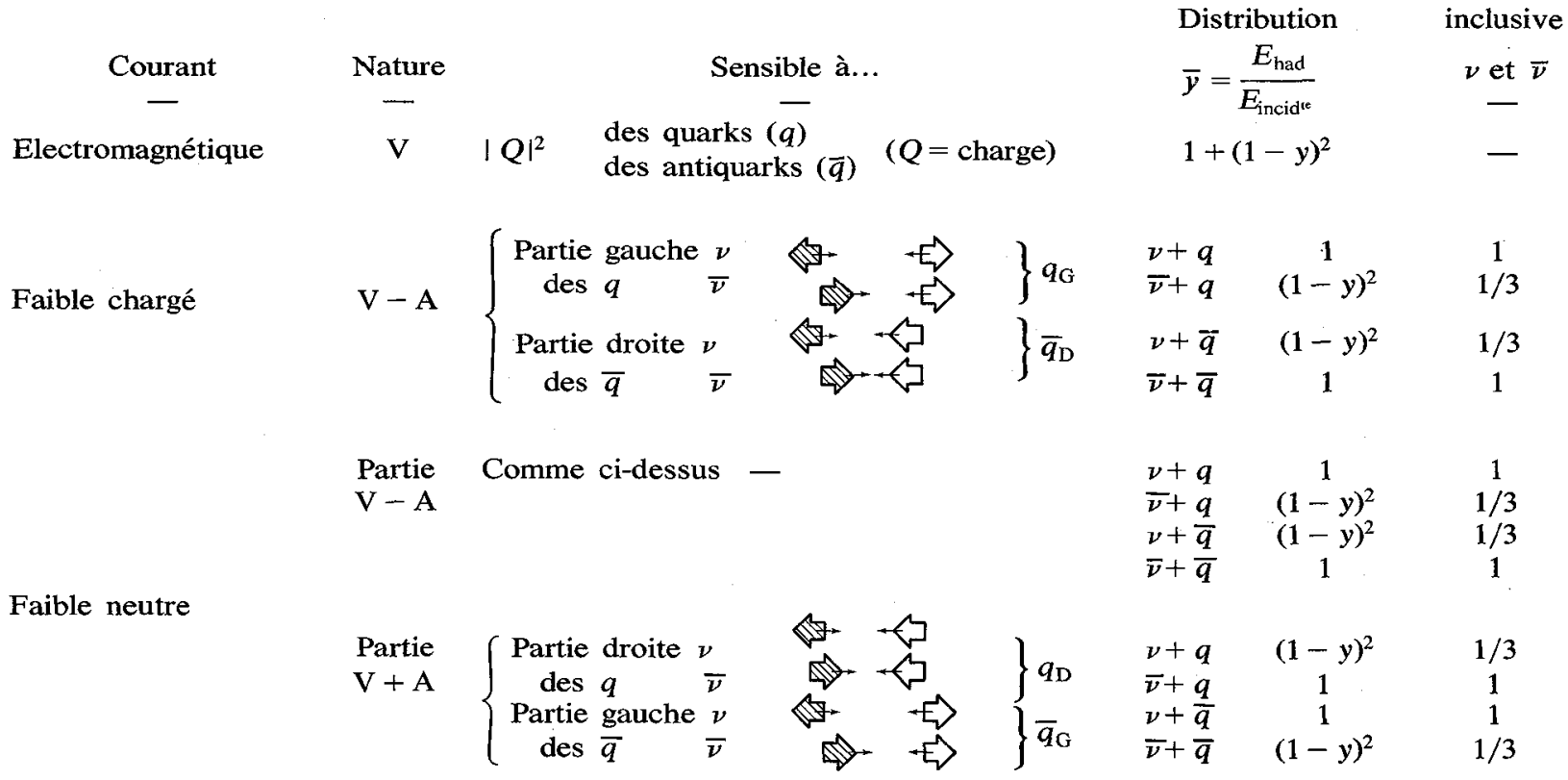

\section{TABLEAU III}

Principaux résultats des expériences sur les réactions inclusives $: \nu_{\mu}\left(\overline{\nu_{\mu}}\right)+N \rightarrow \nu_{\mu}\left(\overline{\nu_{\mu}}\right)+\cdots$

[Experimental results on inclusive reactions: $\nu_{\mu}\left(\bar{\nu}_{\mu}\right)+\mathrm{N} \rightarrow \nu_{\mu}\left(\bar{\nu}_{\mu}\right)+\cdots$ ]

\begin{tabular}{|c|c|c|c|c|c|c|}
\hline $\begin{array}{l}\text { Expérience } \\
\frac{-}{\text { Gargamelle }}\end{array}$ & $\begin{array}{c}\text { Détecteur } \\
\text { (Nature/Masse) } \\
\text { Fréon- } \mathrm{CF}_{3} \mathrm{Br}\end{array}$ & $\begin{array}{l}\text { Faisceau } \\
- \\
\text { CERN/PS }\end{array}$ & $\begin{array}{c}\frac{\sigma(\nu+\mathrm{N} \rightarrow \nu+\cdots)}{\sigma\left(\nu+\mathrm{N} \rightarrow \mu^{-}+\cdots\right)} \\
-\end{array}$ & $\begin{array}{c}\frac{\sigma(\bar{\nu}+\mathrm{N} \rightarrow \bar{\nu}+\cdots)}{\sigma\left(\bar{\nu}+\mathrm{N} \rightarrow \mu^{+}+\cdots\right)} \\
-\end{array}$ & $\begin{array}{c}\text { Parité } \\
\text { Violation }\end{array}$ & $\begin{array}{c}\sin ^{2} \theta_{w} \\
-\end{array}$ \\
\hline & $4,5 \mathrm{t}$ & 1 à $12 \mathrm{GeV}$ & $0,26 \pm 0,04$ & $0,39 \pm 0,06$ & $\frac{\bar{\sigma}_{\mathrm{N}}}{\sigma_{\mathrm{N}}}=0,57 \pm 0,13$ & $0,32 \pm 0,05$ \\
\hline $\begin{array}{l}\text { Harvard } \\
\text { Pennsylvanie } \\
\text { Wisconsin } \\
\text { Fermi Lab. }\end{array}$ & $\begin{array}{c}\text { Scintillateur } \\
\text { liquide } \\
\text { et fer } \\
20 \mathrm{t}\end{array}$ & $\begin{array}{l}\text { Fermi Lab. } \\
\text { Bande large } \\
10-250 \mathrm{GeV}\end{array}$ & $0,29 \pm 0,04$ & $\leqslant 0,39 \pm 0,10$ & $\begin{array}{c}\text { Violation } \\
\frac{\bar{\sigma}_{\mathrm{N}}}{\sigma_{\mathrm{N}}} \leqslant 0,61 \pm 0,25\end{array}$ & 一 \\
\hline $\begin{array}{l}\text { Cal. tech. } \\
\text { Fermi Lab. }\end{array}$ & $\begin{array}{l}\text { Fer } \\
160 \mathrm{t}\end{array}$ & $\begin{array}{c}\text { Fermi Lab. } \\
\text { Dichromatique } \\
\nu_{\pi} \sim 40 \mathrm{GeV} \\
\nu_{\mathrm{K}} \sim 100 \mathrm{GeV}\end{array}$ & $0,24 \pm 0,02$ & $0,34 \pm 0,09$ & Violation & $0,33 \pm 0,05$ \\
\hline $\begin{array}{l}\text { CERN } \\
\text { Dortmund } \\
\text { Heidelberg } \\
\text { Saclay }\end{array}$ & $\begin{array}{l}\text { Fer } \\
900 \mathrm{t}\end{array}$ & $\begin{array}{l}\text { CERN/SPS } \\
\text { Dichromatique } \\
\text { «Parents } \pi \text { et } \\
\text { K de } 200 \mathrm{GeV}\end{array}$ & $0,30 \pm 0,015$ & $0,38 \pm 0,03$ & $\begin{array}{c}\text { Violation } \\
\frac{\bar{\sigma}_{\mathrm{N}}}{\sigma_{\mathrm{N}}}=0,54 \pm 0,06\end{array}$ & $0,26 \pm 0,025$ \\
\hline
\end{tabular}

différence entre les constantes de couplage gauche et droite, mesurée dans Gargamelle et trouvée différente de 0 à 3 écarts-type (voir figure 3), comme sur le rapport $R_{\mathrm{N}}=\bar{\sigma}_{\mathrm{N}} / \sigma_{\mathrm{N}}$, qu'on peut déduire de $\sigma_{\mathrm{N}} / \sigma_{\mathrm{c}}, \bar{\sigma}_{\mathrm{N}} / \bar{\sigma}_{\mathrm{c}}$ et $\bar{\sigma}_{\mathrm{c}} / \sigma_{\mathrm{c}}$.
Dans Gargamelle [18] on obtient $\boldsymbol{R}_{\mathrm{N}}=0,57 \pm 0,13$, la collaboration Harvard-Pennsylvanie-WisconsinFermi lab. (HPWF) [19] trouve $R_{\mathrm{N}} \leqslant 0,61 \pm 0,25$ et l'expérience CDHS [21] donne $R_{\mathrm{N}}=0,54 \pm 0,06$.

La violation de la parité est aujourd'hui bien 


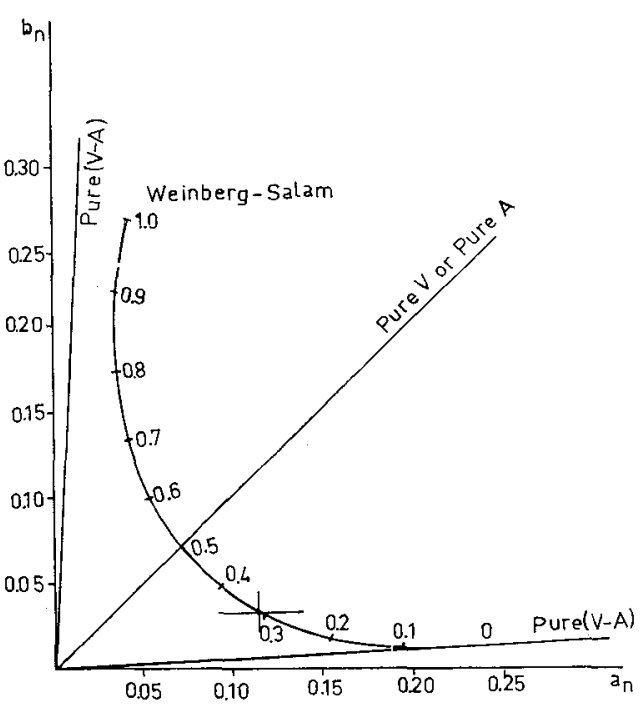

Fig. 3. - Mise en évidence de la violation de la parité dans les processus courants neutres inclusifs dans l'expérience Gargamelle; les quantités $a_{n}$ et $b_{n}$ diffèrent en fait très légèrement des constantes de couplage gauche et droite respectivement, en raison d'une correction due à la faible proportion d'antiquarks dans le nucléon.

[Evidence for parity violation in inclusive neutral current processes, from the Gargamelle experiment; the constants $a_{n}$ and $b_{n}$ are in fact slightly different from the left-handed and right-handed coupling constants, the correction being due to the small fraction of antiquarks in the nucleon.]

établie pour les processus courants neutres, ce qui exclut les modèles de type vectoriel [17]. Ce résultat est complété par les expériences réalisées à Brookhaven sur les canaux élastiques $\nu_{\mu}+\mathrm{p} \rightarrow \nu_{\mu}+\mathrm{p}[22,23]$ et $\bar{\nu}_{\mu}+\mathrm{p} \rightarrow \bar{\nu}_{\mu}+\mathrm{p}[23]$; ces expériences fournissent des données en bon accord en ce qui concerne les neutrinos, et 1'une d'elles [23] permet de mesurer le rapport

$$
R_{\mathrm{e}}=\frac{\sigma\left(\bar{\nu}_{\mu}+\mathrm{p} \rightarrow \bar{\nu}_{\mu}+\mathrm{p}\right)}{\sigma\left(\nu_{\mu}+\mathrm{p} \rightarrow \nu_{\mu}+\mathrm{p}\right)}
$$

qui devrait être égal à 1 si la parité était conservée. Dans un domaine en $q^{2}$ ( $q$ étant la quadri-impulsion transférée) compris entre 0,3 et $0,9(\mathrm{GeV} / \mathrm{c})^{2}$, les auteurs donnent $R_{e}=0,40 \pm 0,20$ où l'erreur est purement statistique; on a encore une indication, quoique moins nette, de violation de la parité. Enfin, on notera que, dans le cadre du modèle de SalamWeinberg, toutes les expériences sur les sections efficaces inclusives ou élastiques fournissent des valeurs de $\sin ^{2} \theta_{\mathrm{w}}$ compatibles entre elles et de l'ordre de 0,3 (voir tableau III).

2.2.2 Structure en spin isotopique du courant neutre. - Dans le cadre du modèle des quarks, la partie non leptonique des courants neutres contient un terme isoscalaire $(\Delta I=0)$, et un terme isovectoriel $(\Delta I=1)[9,10]$. Pour apprécier l'importance relative de chacun de ces termes, il est commode de considérer les canaux de production d'un seul pion selon : $\quad \nu_{\mu}\left(\right.$ ou $\left.\bar{\nu}_{\mu}\right)+\mathrm{N} \rightarrow \nu_{\mu} \quad\left(\right.$ ou $\left.\bar{\nu}_{\mu}\right)+\mathrm{N}+\pi$. Ces réactions sont décrites au moyen d'une amplitude isoscalaire $S$ et de deux amplitudes isovectorielles correspondant aux états respectifs $I=1 / 2\left(V_{1}\right)$ et $I=3 / 2\left(V_{3}\right)$ du système $\pi$-nucléon. Le tableau IV donne l'amplitude de transition pour chacun des 4 canaux possibles en neutrino en fonction de $S, V_{1}$ et $V_{3}$. On voit ainsi que les canaux produisant des pions neutres doivent être deux fois plus fréquents que ceux qui produisent des pions chargés si $V_{3}$ domine, ce résultat étant inversé si $S$ domine. Des résultats déjà publiés de l'expérience Gargamelle [24] fondés sur la comparaison des productions de $\pi^{0}$ et de $\pi^{-}$rendaient peu vraisemblable la dominance de $S$. Très récemment une étude complète des 4 canaux de production d'un pion a pu être réalisée dans Gargamelle remplie d'un mélange léger à base de propane [25]; ce liquide contient des protons libres et les noyaux de carbone sont suffisamment légers pour permettre de séparer les interactions ayant eu lieu sur proton et sur neutron. On peut donc obtenir les sections efficaces sur nucléons libres données dans le tableau IV, à partir d'une méthode de correction nucléaire testée sur plusieurs autres résultats expérimentaux. L'hypothèse d'une amplitude purement isoscalaire est exclue par les données ( $\chi^{2}=30$ à 3 degrés de liberté), mais 1'hypothèse d'une amplitude isovectorielle pure avec isospin final $3 / 2$ est peu vraisemblable $\left(\chi^{2}=10\right.$ à 3 degrés de liberté). On trouve un terme d'interférence différent de 0 à 2 écarts-type ;

$$
\begin{aligned}
& \sigma\left(\nu \mathrm{p} \pi^{9}\right)-\sigma\left(\nu \mathrm{n} \pi^{9}\right)= \\
& =C \cdot R_{\mathrm{e}}\left(\left(2 V_{3}+V_{1}\right)^{*} \cdot S\right)= \\
& =120 \pm 60 \text { en unités arbitraires }) .
\end{aligned}
$$

\section{TABLEAU IV}

Production d'un pion par des neutrinos en courant neutre. Expressions des amplitudes de transition en fonction de $V_{1}$ (amplitude isovectorielle avec état final $I=\frac{1}{2}$ ), $V_{3}$ (amplitude isovectorielle avec état final $I=\frac{3}{2}$ ) et $S$ (amplitude isoscalaire). Sections efficaces mesurées dans l'expérience «neutrinopropane" dans Gargamelle, (en unités arbitraires).

[Single pion production by neutral-current-induced neutrino reactions. Transition amplitudes expressed as functions of $V_{1}$ (isovector amplitude for $I=1 / 2$ final state), $V_{3}$ (isovector amplitude for $I=3 / 2$ final state) and $S$ (isoscalar amplitude). Cross-sections measured in the neutrino-propane Gargamelle experiment, (in arbitrary units).]

$\begin{array}{ccc}\text { Réaction } & \text { Amplitude } & \begin{array}{c}\text { Mesurée } \\ \text { (Unité arbitraire) }\end{array} \\ \nu \mathrm{p} \rightarrow \nu \mathrm{p} \pi^{\circ} & -S+2 V_{3}+V_{\mathrm{t}} & - \\ \nu \mathrm{p} \rightarrow \nu \mathrm{n} \pi^{+} & \sqrt{2\left(S+V_{3}-V_{1}\right)} & 297 \pm 37 \\ \nu \mathrm{n} \rightarrow \nu \mathrm{n} \pi^{\circ} & S+2 V_{3}+V_{1} & 180 \pm 31 \\ \nu \mathrm{n} \rightarrow \nu \mathrm{p} \pi^{-} & \sqrt{2}\left(-S+V_{3}-V_{1}\right) & 237 \pm 59\end{array}$


Dans le plan complexe, on peut délimiter les zones permises pour les extrémités des vecteurs représentatifs de $V_{1}$ et $S, V_{3}$ étant fixé conventionnellement. La figure 4 montre ces zones pour un intervalle de confiance de $68 \%$. Toutefois la distribution de la masse nucléon-pion (en particulier dans le canal $\nu \mathrm{p} \rightarrow \nu \mathrm{p} \pi^{0}$ ) indiquant la présence de $\Delta$, exclut la prépondérance de $V_{1}$ ou de $S$ sur $V_{3}$.

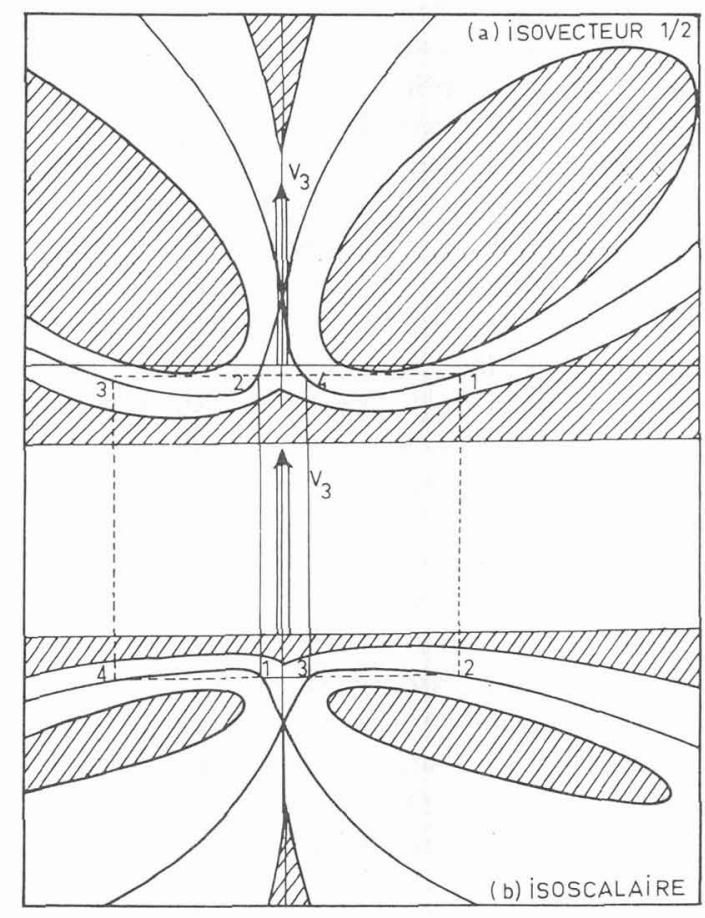

FIG. 4. - Production d'un pion par des courants neutres (expérience neutrino-propane dans Gargamelle). Limites expérimentales à $68 \%$ de confiance dans le plan complexe pour les extrémités des vecteurs représentant: (a) l'amplitude $V_{1}$ (isovecteur avec état final $I=1 / 2$ ); (b) l'amplitude $S$ (isoscalaire). Le vecteur représentant l'amplitude $V_{3}$ (isovecteur avec état final $3 / 2$ ) est fixé conventionnellement en grandeur et direction. Les régions interdites sont hachurées.

[Single pion production by neutral currents (neutrino-propane Gargamelle experiment). Experimental limits (68\% confidence level) in the complex plane for the end points of the vectors representing: (a) the amplitude $V_{1}$ (isovector with $I=1 / 2$ final state); (b) the amplitude $S$ (isoscalar). The vector representing the amplitude $V_{3}$ (isovector with $I=3 / 2$ final state) has been conventionnally fixed in magnitude and direction. Forbidden regions have been shaded.]

2.2.3 Production de particules étranges par les courants neutres. - L'étude des désintégrations de particules étranges n'ayant pas décelé de processus courant neutre au premier ordre de l'interaction faible [3], il était logique de penser que les courants neutres conservent l'étrangeté. Toutefois, il est intéressant de le vérifier directement dans les réactions de neutrinos couvrant un tout autre domaine cinématique que les désintégrations précédentes et faisant intervenir également la partie axiale du courant. Les données de Gargamelle obtenues depuis 1971 tant avec un remplissage de fréon que de propane [26] ont été utilisées pour donner les limites suivantes (à $90 \%$ de confiance) :

$$
\begin{aligned}
& \frac{\sigma_{\mathrm{N}}\left(\Delta S=-1 ; \quad \Lambda^{a} \text { ou } \Sigma^{a} \text { isolé }\right)}{\sigma_{\mathrm{N}}(\text { total })}<1,1 \% \\
& \text { pour les neutrinos , } \\
& \frac{\bar{\sigma}_{\mathrm{N}}\left(\Delta S=-1 ; \Lambda^{\circ} \text { ou } \Sigma^{\circ} \text { isolé }\right)}{\bar{\sigma}_{\mathrm{N}}(\text { total })}<0,51 \%
\end{aligned}
$$

On a également mesuré le taux de production de particules étranges en paires (en supposant la conservation de l'étrangeté) tout au moins quand l'une de ces particules donnait une topologie de type $\mathrm{V}^{0}\left(\Lambda^{0}, \Sigma^{0}, \mathrm{~K}^{0}\right.$ ou $\left.\overline{\mathrm{K}}^{0}\right)$. On a trouvé :

$$
\begin{aligned}
& \frac{\bar{\sigma}_{\mathrm{N}}\left(\Delta S=0 ; \Lambda^{0}, \Sigma^{0}, \mathrm{~K}^{0}, \overline{\mathrm{K}}^{0}\right)}{\bar{\sigma}_{\mathrm{N}} \text { (total) }}=\left(\begin{array}{r}
+0,9 \\
-0,8
\end{array}\right) \% \\
& \frac{\bar{\sigma}_{\mathrm{N}}\left(\Delta S=0 ; \quad \Lambda^{0}, \Sigma^{0}, \mathrm{~K}^{0}, \overline{\mathrm{K}}^{0}\right)}{\bar{\sigma}_{\mathrm{N}} \text { (total) }}=\left(1,3 \begin{array}{r}
+1,2 \\
-0,7
\end{array}\right) \% .
\end{aligned}
$$

Une étude des mécanismes de production de ces particules étranges par des courants neutres, à partir de sections efficaces obtenues en neutrino et en antineutrino permet de donner une limite supérieure pour la contribution des quarks étranges (paires $\lambda \bar{\lambda}$ ) du nucléon égale à $4 \%$ (90\% de confiance) de la contribution des quarks $p$ et $n$ [26].

2. 3 CONCLUSION SUR LES COURANTS NEUTRES. - Les résultats précédents permettent les conclusions suivantes :

a) Toutes les expériences confirment la violation de la parité dans les courants neutres.

b) En ce qui concerne la structure en isospin, les données de Gargamelle sur la production d'un pion, tout en confirmant l'importance du terme isovecteur, indiquent une contribution appréciable du terme isoscalaire.

c) La conservation de l'étrangeté, prédite par le mécanisme de Glashow-Iliopoulos-Maiani [4] est vérifiée au niveau de l'ordre de quelques $10^{-3}$.

d) Tous les résultats expérimentaux sont en accord avec les prédictions du modèle de SalamWeinberg avec $\sin ^{2} \theta_{\mathrm{W}} \sim 0,3$.

Des résultats plus précis devraient intervenir dans des délais assez rapprochés :

a) L'étude des distributions de la variable $y$ de Bjorken définie plus haut est possible en faisceau dichromatique où l'énergie du neutrino est connue. Elle constituera un test important de la structure en espace-temps des courants neutres, et par suite du modèle de Salam-Weinberg.

b) L'étude des sections efficaces inclusives (et des distributions en y) mesurées séparément dans les réactions sur proton $(\nu+\mathrm{p})$ et sur neutron $(\nu+\mathrm{n})$ permettra également de préciser la structure en spin isotopique. 
c) L'invariance d'échelle, suggérée par la constance avec l'énergie des rapports $\sigma_{\mathrm{N}} / \sigma_{\mathrm{c}}$ et $\bar{\sigma}_{\mathrm{N}} / \bar{\sigma}_{\mathrm{c}}$, pourra être testée de manière plus précise dans un faisceau dichromatique.

d) Enfin l'augmentation de la statistique en processus purement leptoniques $\left(\nu_{\mu}+\mathrm{e}^{-}\right.$ou $\left.\bar{\nu}_{\mu}+\mathrm{e}^{-}\right)$ fournira un test très direct des différentes théories de jauge envisagées.

3. Effets dus à la production de « charme » par les neutrinos. - Dans le mécanisme de Glashow, Iliopoulos et Maiani, la conservation de l'étrangeté par les courants neutres s'explique par l'introduction de nouveaux courants faibles chargés couplant les quarks $\mathrm{n}$ et $\lambda$ à un quatrième quark $\mathbf{p}^{\prime}$ portant un nouveau nombre quantique : le charme [4]. Les réactions de neutrino induites par ces nouveaux courants chargés peuvent ainsi produire une particule charmée à partir des quarks habituels du nucléon. Nous rappellerons d'abord les différentes signatures de ces nouvelles réactions, puis nous examinerons leurs mises en évidence expérimentales.

\subsection{SIGNATURE DE LA PRODUCTION DE CHARME.} - Les nouveaux courants introduits par Glashow, Iliopoulos et Maiani couplent d'une part le quark $\mathrm{n}$ et le quark $p^{\prime}$ avec un facteur $\sin \theta_{\mathrm{c}}$ (où $\theta_{\mathrm{c}}$ est l'angle de Cabibbo [27]) et d'autre part le quark étrange $\lambda$ et le quark p' avec un facteur $\cos \theta_{\mathrm{c}}$ [4]. Les réactions de production à partir des quarks du nucléon sont les suivantes :

$$
\left\{\begin{array}{c}
\nu+\mathrm{n} \rightarrow \mu^{-}+\mathrm{p}^{\prime} \\
\nu+\lambda \rightarrow \mu^{-}+\mathrm{p}^{\prime} \\
\bar{\nu}+\bar{\lambda} \rightarrow \mu^{+}+\overline{\mathbf{p}}^{\prime} \\
\bar{\nu}+\overline{\mathbf{n}} \rightarrow \mu^{+}+\overline{\mathbf{p}}^{\prime}
\end{array}\right.
$$

Seule la première peut avoir lieu sur un quark de valence, toutes les autres concernant un quark ou antiquark de la mer dont la contribution dans les réactions $\nu+N$ ou $\bar{\nu}+\mathrm{N}$ est faible ; en revanche, le premier processus est défavorisé par rapport aux deux suivants par le couplage en $\sin \theta_{c}$.

La désintégration des particules charmées produites śe fait évidemment préférentiellement grâce au courant couplant $\mathrm{p}^{\prime}$ et $\lambda$, à cause du facteur en $\cos \theta_{\mathrm{c}}$; (processus $\Delta C=\Delta Q$ ). On a ainsi, pour une désintégration semi-leptonique :

$$
\begin{array}{ll}
p^{\prime} \rightarrow \lambda \mu^{+} \nu_{\mu} & \left(\text { ou } \lambda \mathrm{e}^{+} \nu_{\mathrm{e}}\right) \\
\bar{p}^{\prime} \rightarrow \bar{\lambda} \mu^{-} \bar{\nu}_{\mu} & \left(\text { ou } \bar{\lambda} \mathrm{e}^{-} \bar{\nu}_{\mathrm{e}}\right) .
\end{array}
$$

Par ailleurs, les ordres de grandeur des temps de vie et masses des nouvelles particules conduisent à des parcours trop faibles pour être observés dans les détecteurs habituels, sauf en émulsion.

La figure 5 montre le résultat apparent de la production suivie de la désintégration semi-leptoni-
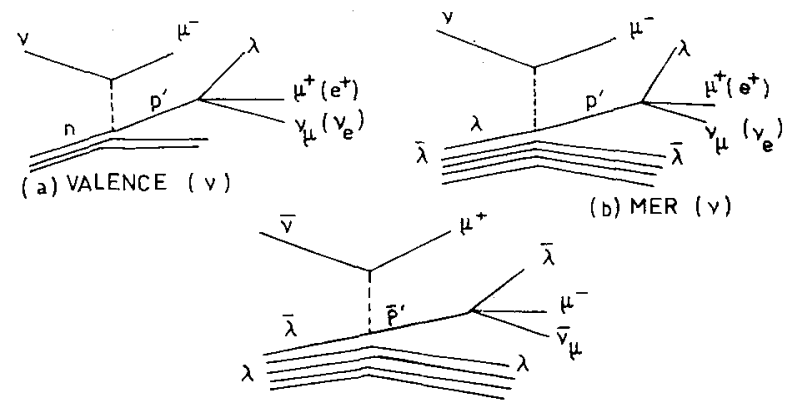

(b) $\operatorname{MER}(v)$

(c) MER (v)

FIG. 5. - Signature de la production de charme par des neutrinos $(a, b)$ ou des antineutrinos $(c)$. Le processus $(a)$ est dû aux quarks de valence, les processus $(b)$ et $(c)$ aux quarks ou antiquarks de la mer.

[Signature of charmed particle production by neutrinos $(a, b)$ or antineutrinos $(c)$. Process $(a)$ is due to valence quarks, whereas processes (b) and (c) are due to sea quarks or antiquarks.]

que des objets charmés; dans tous les cas on observera deux leptons chargés de signes opposés ( $\mu^{+} \mu^{-}$ou $\mu^{-} \mathrm{e}^{+}$ou $\left.\mu^{+} \mathrm{e}^{-}\right)$et une ou deux particules étranges, notamment sous forme de $\mathrm{V}^{0}$. Nous appellerons lepton de bon signe celui qui est directement produit par le neutrino incident, et lepton de mauvais signe celui qui provient de la particule charmée. Enfin, lorsque la désintégration de la particule charmée se fait selon un mode non leptonique la particule étrange finale aura l'étrangeté $S=-1$ en neutrino et $S=+1$ en antineutrino, ce qui constitue une violation apparente de la règle $\Delta S=\Delta Q[17]$.

3.2 EXPÉRIENCES OBSERVANT DEUX LEPTONS DE SIGNES OPPOSÉS DANS L'ÉTAT FINAL SANS CONNÂ̂TRE LES DÉTAILS DU SYSTÈME HADRONIQUE ASSOCIÉ. - Les expériences les plus simples consistent à observer les événements à 2 leptons finals de signes opposés (essentiellement à 2 muons finals), sans se préoccuper de la nature des hadrons produits avec eux $[28,29,30,31]$. Un premier problème expérimental est lié au critère d'acceptance des muons, et à la correction correspondante. Celle-ci est généralement importante pour le muon de mauvais signe qui peut être assez lent pour ne pas être identifié, son énergie étant de l'ordre de celle d'un hadron. Surtout, cette correction dépend du modèle de production de charme. Aussi, les taux de production présentés dans le tableau $V$ ne sont-ils pas directement comparables, car ils représentent la fraction d'événements courant chargé à 2 muons dans des critères d'acceptance variant d'une expérience à l'autre. En outre, certaines expériences ne connaissent pas toujours le signe des muons [28, 30]. Enfin toutes sont sujettes au bruit de fond dû à la désintégration de $\pi$ et de $\mathrm{K}$ en vol $(\pi \rightarrow \mu \nu$ et $\mathrm{K} \rightarrow \mu \nu$ ) simulant un second muon ; l'estimation de ce bruit de fond est délicate car elle nécessite la connaissance de la gerbe hadronique (multiplicité, distribution en énergie des hadrons, proportions de 


\section{TABLEAU V}

Principaux résultats des expériences observant des dimuons de signes opposés ; (expériences d'électronique).

[Experimental results on opposite sign dimuon events ; (electronic experiments).]

\begin{tabular}{|c|c|c|c|c|c|c|}
\hline Expérience & $\begin{array}{c}\text { Masse } \\
\text { Détecteur }\end{array}$ & Faisceau & $\begin{array}{l}\text { Signe des } \\
2 \mu \text { connu? }\end{array}$ & $\begin{array}{l}\text { Nombre de } \\
\text { cand. } \mu^{+} \mu^{-}\end{array}$ & $\begin{array}{l}\text { Bruit de } \\
\text { fond }\end{array}$ & $\begin{array}{c}\text { Taux } \\
\mu^{+} \mu^{-/ C o u r a n t s} \\
\text { chargés }\end{array}$ \\
\hline Serpoukhov & $\overline{34 \mathrm{t}}$ & 2 à $25 \mathrm{GeV}$ & $\overline{\mathrm{NON}}$ & $40 \pm 10$ & $-\overline{50} \%$ & $1,6_{-0,8}^{+1,2} \%$ \\
\hline $\begin{array}{l}\text { Harvard } \\
\text { Pennsylvanie } \\
\text { Wisconsin } \\
\text { Fermi Lab. }\end{array}$ & $\begin{array}{l}60 \mathrm{t} \\
\text { puis } \\
400 \mathrm{t}\end{array}$ & $\begin{array}{c}\stackrel{\nu}{100}-250 \mathrm{GeV} \\
\bar{\nu} \\
100-200 \mathrm{GeV}\end{array}$ & OUI & 42 & $\leqslant 20 \%$ & $0,8 \pm 0,3 \%$ \\
\hline $\begin{array}{l}\text { Cal. tech. } \\
\text { Fermi Lab. }\end{array}$ & $160 t$ & $\begin{array}{l}\text { Fermi Lab. } \nu \\
\text { Dichromatique } \\
\sim 150 \mathrm{GeV} \\
\qquad \bar{\nu}\end{array}$ & $\begin{array}{l}\begin{array}{c}\text { seulement } \\
\text { pour }\end{array} \\
21 \text { Evts. }\end{array}$ & $\begin{array}{l}62 \\
30\end{array}$ & $\sim 20 \%$ & $\begin{array}{c}\sim 1 \text { à } 2 \% \\
\text { de } 50 \text { à } \\
200 \mathrm{GeV}\end{array}$ \\
\hline $\begin{array}{l}\text { CERN } \\
\text { Dortmund } \\
\text { Heidelberg } \\
\text { Saclay }\end{array}$ & $900 \mathrm{t}$ & $\begin{array}{l}\text { CERN/SPS } \nu \\
\text { Dichromatique } \\
\text { Parents : } 200 \mathrm{GeV} \\
\quad \bar{\nu}\end{array}$ & OUI & 257 & $14 \%$ & $\sim 0,5 \%$ \\
\hline
\end{tabular}

$\pi$ et de $\mathrm{K}$ dans la gerbe, longueurs d'interaction des $\pi$ et des $\mathrm{K}$, etc...). Le tableau $\mathrm{V}$ montre que le taux de production de dimuons est de l'ordre de $10^{-3}$ à $10^{-2}$. De plus, quand les signes des $\mu$ sont connus, on observe toujours la dominance énergétique du $\mu$ de bon signe $[29,31]$ et une forte corrélation angulaire entre le $\mu$ de mauvais signe et les hadrons [29, 31], en accord avec l'interprétation du charme. La figure 6 illustre le premier effet à partir des données de l'expérience CDHS au SPS du CERN. Cette dernière expérience, réalisée en faisceau dichromatique a pu en outre estimer à $(8 \pm 4) \%$ la fraction de l'énergie emportée par le neutrino de l'état final, ce qui confirme la limite supérieure de 15 à $20 \%$ donnée antérieurement par Cal. Tech. [30].

\subsection{EXPÉRIENCES OBSERVANT DES DILEPTONS} $\mu^{-} \mathrm{e}^{+}$OU $\mu^{+} \mathrm{e}^{-}$ASSOCIÉS A DES PARTICULES ÉTRAN-

$E$ ( $\mu$ de "mauvais" signe)

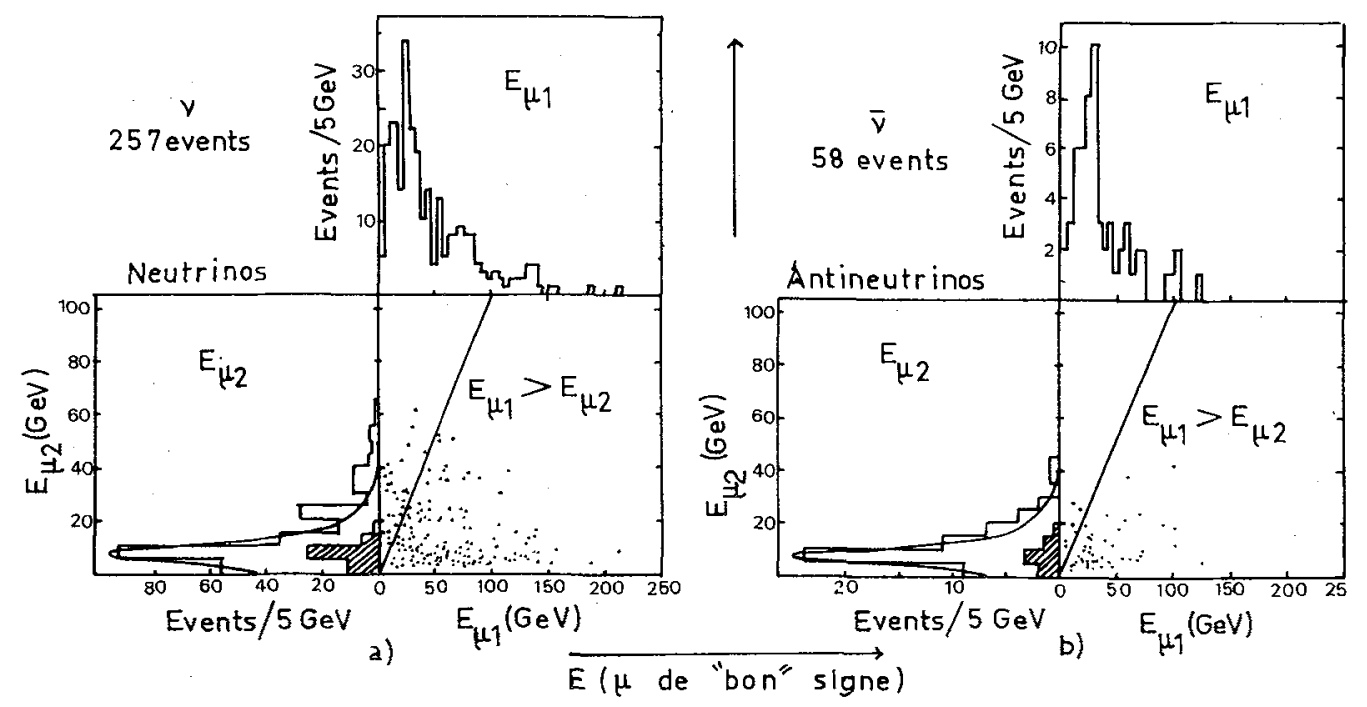

Fig. 6. - Evénements à deux muons de signes opposés, (collaboration CERN - Dortmund - Heidelberg - Saclay). Diagramme représentant l'énergie du $\mu$ de bon signe $\left(E \mu_{1}\right)$ par rapport à l'énergie de l'autre $\mu\left(E \mu_{2}\right)$.

[Opposite sign dimuon events, (CERN - Dortmund - Heidelberg - Saclay collaboration). Scatter plot of $E \mu_{1}$ (energy of the ordinary muon) versus $E \mu_{2}$ (energy of the wrong sign muon).] 
GES. - Une autre série de résultats provient des chambres à bulles remplies d'un liquide à faible longueur de radiation (fréon pour «Gargamelle », mélanges d'hydrogène et de néon pour la chambre de 15 pieds de Fermi Lab.) permettant d'identifier les électrons et positrons. On détecte donc des

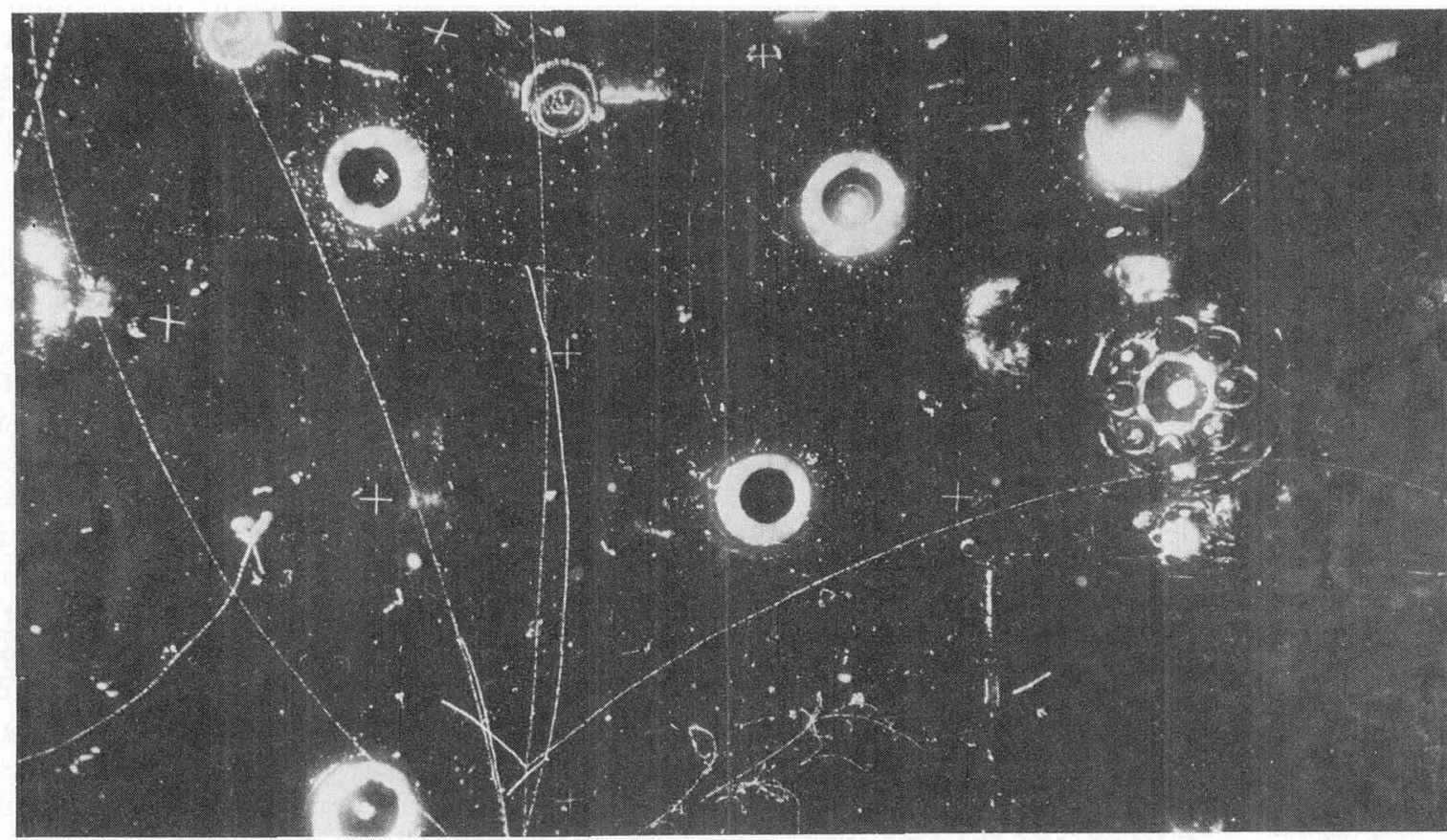

Fig. 7. - Evénement candidat pour la production d'une particule charmée dans Gargamelle. On notera : le $\mu^{-}$(particùle négative sortant de la chambre) le positron (caractérisé par son bremsstrahlung) et le $\mathrm{V}^{0}$ associé. (Les particules positives sont courbées vers la gauche).

[A candidate for charmed particle production in Gargamelle. One can identify the $\mu^{-}$(negative track leaving the chamber), the positron (characterized by its bremsstrahlung) and the associated $\mathrm{V}^{0}$. (Left-handed curvature corresponds to positive particles).]

\section{TABLEAU VI}

Principaux résultats des expériences observant des dileptons ue associés à des particules étranges; (expériences de chambre à bulles).

[Experimental results on $\mu \mathrm{e}$ dilepton events associated with strange particles; (bubble chamber experiments).]

\begin{tabular}{|c|c|c|c|c|c|c|}
\hline Experie & iscea & $\begin{array}{l}\text { Energie } \mathrm{e}^{ \pm} \\
\text {supérieure à : }\end{array}$ & $\begin{array}{l}\mathrm{Nb} \text { de candidats } \\
\mu \mathrm{e} \text { (et bruit de } \\
\text { fond) }\end{array}$ & $\begin{array}{c}\text { Candidats } \\
\mu \mathrm{e}+\mathrm{V}^{\circ}\end{array}$ & $\begin{array}{c}\text { Nombre moyen } \\
\text { de particules } \\
\text { étranges } \\
\text { neutres/evt. }\end{array}$ & $\begin{array}{c}\text { Taux } \\
\mu \text { /Courants } \\
\text { charges }\end{array}$ \\
\hline$\overline{\text { gamelle }}$ & $\bar{\nu}$ & $200 \overline{\mathrm{MeV}}$ & $20 u^{-} \overline{e^{+}}(6,2)$ & $\overline{3}$ & - & $(0.31 \pm 0.13) \%$ \\
\hline
\end{tabular}

1 à $12 \mathrm{GeV}$

Berkeley

CERN

Hawaï

$10-200 \mathrm{GeV}$

$800 \mathrm{MeV}$

$17 \mu^{-} \mathrm{e}^{+}(1)$

11

$\left(\mathrm{K}^{\circ}\right)$

Wisconsin

(Ch. de 15)

Columbia

Brookhaven

(Ch. de 15')

$$
\nu
$$

$10-200 \mathrm{GeV} \quad 300 \mathrm{MeV} \quad 81 \mu^{-} \mathrm{e}^{+}(12)$

15

$$
\dot{0}, 6 \pm 0,2
$$

$(0,50 \pm 0,15) \%$

Fermi Lab.

Serpoukhov

Moscou

$$
\bar{\nu}
$$

Michigan

$10-150 \mathrm{GeV}$

$200 \mathrm{MeV}$

$1 \mu^{+} e^{-}(0,2)$

0

$<0,5 \%$

(90\% confiance)

F. 82 
dileptons $\mu^{-} \mathrm{e}^{+}$ou $\mu^{+} \mathrm{e}^{-}$, le muon étant identifié à haute énergie par un détecteur externe ou E.M.I. (External Muon Identifier). La figure 7 montre l'un des premiers de ces événements, trouvé dans Gargamelle [32]. Les taux de dileptons indiqués dans le tableau VI $[32,33,34,35]$ doivent encore être corrigés, les $\mathrm{e}^{ \pm}$n'étant identifiés clairement qu'au-dessus d'une certaine énergie ; les ordres de grandeur sont compatibles avec ceux des expériences décrites plus haut. La caractéristique principale de ces expériences est qu'elles peuvent observer les $\mathrm{V}^{0}$ associés à ces dileptons. (Fig. 7). Toutes les expériences réalisées avec des neutrinos observent une très forte corrélation entre les dileptons et la présence d'un $\mathrm{V}^{0}$. Le nombre moyen de particules étranges associées est encore controversé, la collaboration Berkeley-CERN-Hawai-Wisconsin [33] trouvant $(1,84+0,63) \mathrm{K}^{0}$ par événement, alors que la collaboration Columbia-Brookhaven [34] ne trouve que $(0,6 \pm 0,2)$ particule étrange neutre par événement.

3.4 AUTRES INDICES DE PRODUCTION DE CHARME. - La violation apparente de la règle $\Delta S=\Delta Q$, simulée par la désintégration d'une particule charmée a été observée dans la chambre à bulles à hydrogène de 7 pieds de Brookhaven en 1975 [36] à partir d'un événement s'interprétant préférentiellement selon la réaction :

$$
\nu_{\mu}+\mathrm{p} \rightarrow \mu^{-}+\Lambda^{0}+\pi^{+}+\pi^{+}+\pi^{+}+\pi^{-} .
$$

La crédibilité de cette interprétation s'est accrue lors de la découverte de l'antibaryon charmé $\overline{\Lambda_{\mathrm{c}}^{+}}$ en photoproduction à Fermi Lab. [8] $\left(M\left(\Lambda_{\mathrm{c}}^{+}\right)=2,26 \pm 0,01 \mathrm{GeV} / c^{2}\right)$ car la masse de l'une des combinaisons $\left(\Lambda^{0} \pi^{+} \pi^{+} \pi^{-}\right)$est précisément $(2,260 \pm 0,009) \mathrm{GeV} / c^{2}$. La collaboration Fermi Lab.-Michigan [37] a donné la limite supérieure suivante :

$$
\frac{\sigma(\Delta S=-\Delta Q)}{\sigma\left(\nu+\mathrm{p} \rightarrow \mu^{-}+\cdots\right)}<3,6 \% \text { (à } 90 \% \text { de confiance) },
$$

à partir d'une expérience réalisée dans la chambre à bulles de 15 pieds remplie d'hydrogène. Un résultat plus net a été obtenu dans Gargamelle [32] à partir des événements présentant un $\Lambda^{0}$ sans autre particule étrange détectée; sur un total de 106 événements, seulement $64 \pm 18$ peuvent être expliqués par une production associée avec une particule étrange non détectée. On a donc un excès de $42 \pm 20$ événements du type $\Delta S=-\Delta Q$. En comparant le taux de ce type d'événements aux taux de dileptons, on a pu estimer le rapport d'embranchement des modes semi-leptoniques aux modes non leptoniques :

$B=\frac{\Gamma\left(C \rightarrow\left(\Lambda \mathrm{e}^{+} \nu_{. .}\right) \text {et }\left(\Lambda \mu^{+} \nu_{\ldots} .\right)\right.}{\Gamma(C \rightarrow \Lambda+\text { hadrons })}=(15+18) \%$
Enfin signalons qu'une expérience réalisée à Fermi Lab. avec des émulsions et un dispositif de chambres à étincelles [38] a pu associer un $V^{0}$ détecté par ces dernières à une interaction dans l'émulsion présentant une trace de $182 \mu$ se désintégrant très vraisemblablement ; s'il s'agit d'une particule charmée, on a une estimation de son temps de vie: environ $6 \times 10^{-13} \mathrm{~s}$.

\subsection{CONCLUSION SUR LA PRODUCTION DE} CHARME. - Les différentes signatures prévues pour la production de particules charmées ont été observées. Les dileptons de signes opposés s'interprètent bien par l'hypothèse du charme en raison de la forte corrélation entre le lepton de mauvais signe et les hadrons et surtout en raison de leur association avec les particules étranges, clairement mis en évidence par diverses expériences. La détermination des taux de production reste encore imprécise, en raison des coupures expérimentales délicates à corriger et des problèmes de bruit de fond. Le mécanisme de production reste à approfondir; en particulier il conviendra de séparer la contribution des quarks de valence et des quarks de la mer pour les réactions de neutrino. Il sera également intéressant d'identifier les particules charmées produites, par exemple en les observant dans des spectres de masse invariante à partir de leurs modes non leptoniques.

4. Le point sur quelques effets controversés à très haute énergie. - Les résultats expérimentaux présentés ci-dessus s'inscrivent très bien dans le cadre théorique d'un modèle standard à 4 quarks et 4 leptons [39]. Toutefois, on a signalé quelques effets, encore controversés, dont l'interprétation pourrait nécessiter de nouveaux quarks ou leptons, donc de nouveaux nombres quantiques. Nous passerons successivement en revue les dileptons de même signe et les trimuons, puis les anomalies à grand $y$; tous ces effets concernent les processus courants chargés.

4.1 DILEPTONS DE MÊME SIGNE ET TRIMUONS. Les expériences ayant mis en évidence des dileptons de signes opposés ont enregistré également des événements comportant deux leptons de même: signe $[29,34,31]$ et même quelques événements à 3 muons $[40,30,31]$. Le problème crucial est évidemment l'évaluation du bruit de fond dû surtout aux désintégrations $\pi \rightarrow \mu \nu$ et $\mathrm{K} \rightarrow \mu \nu$ en vol, simulant la présence d'un muon supplémentaire. On notera sur le tableau VII qu'aucun signal en dileptons de même signe n'est à plus de deux écarts-type de 0 , et que le bruit de fond est du même ordre de grandeur que le signal. On peut donc conclure que si l'effet est réel, il est peu important ; à ce niveau, on peut trouver des explications dans le cadre du modèle standard, (par exemple la production d'une paire de particules charmées). 


\section{TABLEAU VII}

Principaux résultats expérimentaux sur la production de dileptons de même signe.

[Experimental results on like-sign dilepton events.]

\begin{tabular}{|c|c|c|}
\hline $\begin{array}{c}\text { Expériences } \\
-\end{array}$ & $\begin{array}{l}\text { Nb de candidats } \\
\text { (et bruit de fond) } \\
\text { _ }\end{array}$ & $\left.\begin{array}{l}\mathrm{ou} \\
+\mu^{+}\end{array}\right) /\left(\mu^{-} \mu^{*}\right.$ \\
\hline $\begin{array}{l}\text { Harvard } \\
\text { Pennsylvanie } \\
\text { Rutgers } \\
\text { Fermi Lab. }\end{array}$ & $\begin{array}{l}7 \mu^{-} \mu^{-} \text {en } \nu \\
3 \mu^{+} \mu^{+} \text {en } \bar{\nu}\end{array}$ & $0,10 \pm 0,05$ \\
\hline $\begin{array}{l}\text { Columbia } \\
\text { Brookhaven } \\
\text { (Ch. à Bulles } \\
\left.\text { de } 15^{\prime}\right)\end{array}$ & $\begin{array}{l}\quad 6 \mu \mathrm{e}^{-} \\
\text {(bruit de fond : 3) }\end{array}$ & $\begin{array}{c}0,2 \pm 0,1 \\
\left(E_{\mathrm{e}}>1 \mathrm{GeV}\right. \\
\left.E_{\mu}>10 \mathrm{GeV}\right)\end{array}$ \\
\hline $\begin{array}{l}\text { CERN } \\
\text { Dortmund } \\
\text { Heidelberg } \\
\text { Saclay }\end{array}$ & $\begin{array}{c}31 \mu^{-} \mu^{-} \text {en } \nu \\
\text { (bruit de fond : } 21 \text { ) } \\
5 \mu^{+} \mu^{+} \text {en } \bar{\nu} \\
\text { (bruit de fond : } 2,2 \text { ) }\end{array}$ & $\begin{aligned} & \leqslant 5 \% \\
\left(E_{\mu}\right. & >5 \mathrm{GeV})\end{aligned}$ \\
\hline
\end{tabular}

En ce qui concerne la faible statistique observée en trimuons, les conclusions expérimentales sont contradictoires. Elles sont résumées dans le tableau VIII. L'explication par du bruit de fond, exclue par la collaboration Harvard-Pennsylvanie-WisconsinRutgers-Fermi Lab. (HPWRF) [40], de même que par la collaboration Cal. Tech.-Fermi Lab. [30], est considérée cependant comme plausible par la collaboration CDHS [31]. Par ailleurs, l'expérience de Cal. Tech.-Fermi Lab. [30] retient l'interprétation par la production d'une paire $\mu^{+} \mu^{-}$au vertex hadronique, alors que la collaboration HPWRF [40] l'exclut, en même temps que tout phénomène hadronique; ces derniers auteurs envisagent l'introduction de nouveaux leptons [41]. On peut spéculer sur les différences d'appareillage, notamment de faisceaux (faisceau mélangé de $\nu_{\mu}$ et $\bar{\nu}_{\mu}$ dans le rapport de 5 à 1 et dans une large bande d'énergie pour

\section{TABLEAU VIII}

Principaux résultats expérimentaux sur la production de trimuons.

[Experimental results on trimuon events.]

Expériences
Harvard
Pennsylvanie
Wisconsin
Rutgers
Fermi Lab.

Cal, tech. Fermi Lab.

CERN

Dortmund Heidelberg Saclay $\begin{array}{cc}\begin{array}{c}\text { Nb de candidats } \\ \text { (et bruit de fond) }\end{array} & \text { Interprétation } \\ - & - \\ 6 \mu^{-} \mu^{-} \mu^{+} & \frac{\mu \mu \mu}{\mu^{+} \mu^{-}+\cdots} \underset{E>100 \mathrm{GeV}}{\sim 5 \% \text { pour }}\end{array}$

Leptons lourds ?

$\begin{array}{cc}2 & \frac{\mu \mu \mu}{\mu+\cdots} \sim 3 \times 10^{-4} \\ \text { (bruit de fond : } 0,08) & \begin{array}{c}\text { Paire } \mu^{+} \mu^{-} \text {produite au } \\ \text { vertex hadronique ? }\end{array}\end{array}$

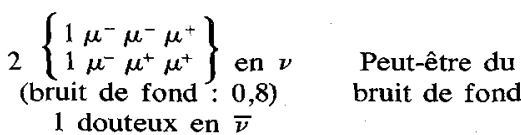

HPWRF, faisceau dichromatique de neutrinos pour Cal. Tech.-Fermi Lab., faisceau dichromatique en $\nu_{\mu}$ comme en $\bar{\nu}_{\mu}$, pour CDHS). L'expérience CDHS doit se poursuivre avec le faisceau à large bande d'énergie du SPS ; par ailleurs l'expérience de Cal. Tech.-Fermi Lab. dispose maintenant d'un détecteur à double cible permettant une mesure du bruit de fond. La situation expérimentale devrait donc se clarifier prochainement.

4.2 LES ANOMALIES A GRAND Y DANS LES PROCESSUS COURANTS CHARGES. - Le tableau II montre le lien entre la structure $\mathrm{V}-\mathrm{A}$ du courant faible chargé et la composition du nucléon en quarks et en antiquarks. A des énergies inférieures à $50 \mathrm{GeV}$ toutes les données sont en accord avec l'image d'un nucléon formé de 3 quarks de valence et d'un peu de mer de même qu'avec un courant faible $\mathrm{V}-\mathrm{A}$ [42]. On doit alors observer une distribution en $y$ de la forme [42] :

$$
\begin{aligned}
& \frac{\mathrm{d} \sigma_{\mathrm{c}}}{\mathrm{d} y}=C\left[(1+B)+(1-B)(1-y)^{2}\right] \\
& \frac{\mathrm{d} \bar{\sigma}_{\mathrm{c}}}{\mathrm{d} y}=\bar{C}\left[(1+\bar{B})(1-y)^{2}+1-\bar{B}\right]
\end{aligned}
$$

où $B \approx \bar{B}$ s'interprète comme le rapport de la contribution des quarks de valence à celle de l'ensemble de tous les quarks et antiquarks du nucléon; ( $B=\bar{B}=1$ si l'on néglige la mer). En raison d'une violation de l'invariance d'échelle prévue dans les théories de liberté asymptotique, on prédit [43] une très légère diminution de $B$ et $\bar{B}$ avec l'énergie et par suite une très légère augmentation du rapport $\bar{\sigma}_{c} / \sigma_{c}$ des sections efficaces inclusives à partir de sa valeur de basse énergie, proche de $1 / 3$. Or, la collaboration Harvard-Pennsylvanie-Wisconsin-Fermi Lab. (HPWF) avait signalé deux gros effects inattendus au-dessus de $30 \mathrm{GeV}$ :

a) une anomalie à grand y [44] dans les antineutrinos se traduisant par $\bar{B}=0,41 \pm 0,13$ nettement inférieur à 1 et surtout à $B(B=0,83 \pm 0,20)$;

b) une importante remontée [45] du rapport des sections efficaces $\bar{\sigma}_{\mathrm{c}} / \sigma_{\mathrm{c}}$ jusqu'à des valeurs de l'ordre de 0,6 à 0,7 .

Il y a un an, l'expérience de Cal. Tech.-Fermi Lab. n'observait pas d'effet dans les sections efficaces [46] mais n'excluait pas une anomalie en $y$ en antineutrino [47], tandis que la collaboration Fermi Lab.-Serpoukhov-Moscou-Michigan, utilisant la chambre à bulle de 15 pieds, ne confirmait pas l'existence de cette anomalie [48]. Très récemment, trois résultats ont pratiquement exclu l'anomalie en $y$ de même que la remontée du rapport $\bar{\sigma}_{\mathrm{c}} / \sigma_{\mathrm{c}}$; il s'agit des nouvelles données de Cal. Tech.-Fermi Lab. [49], de résultats préliminaires de la chambre à bulles BEBC exposée au faisceau dichromatique du SPS du CERN [50] et de l'expérience CDHS dans le même faisceau [51]. Les figures 8 et 9 , tirées de cette dernière expérience, ne montrent pas de varia- 


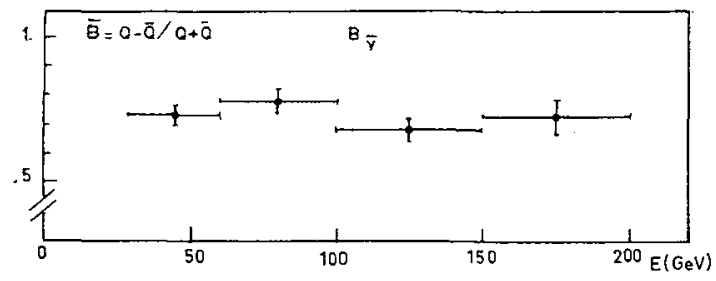

FIG. 8. - Absence d'anomalie en $y$. Le rapport $\bar{B}$ pour les réactions d'antineutrino en fonction de l'énergie incidente (expérience CERN-Dortmund-Heidelberg-Saclay).

[Absence of $y$ anomaly. The ratio $\bar{B}$ for antineutrino reactions as a function of the incident energy; (CERN-Dortmund-HeidelbergSaclay experiment).]

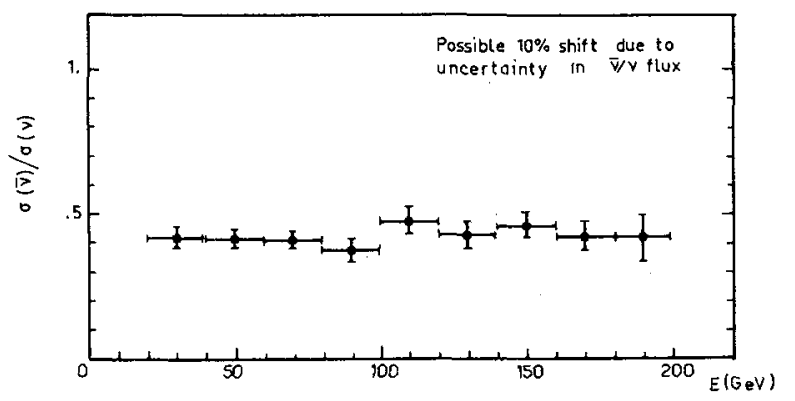

FrG. 9. - Absence d'anomalie dans les sections efficaces. Le rapport $\bar{\sigma}_{\mathrm{c}} / \sigma_{\mathrm{c}}$ en fonction de l'énergie; (expérience CERN-Dortmund-Heidelberg-Saclay).

[Absence of cross-section anomaly. The ratio $\bar{\sigma}_{\mathrm{c}} / \sigma_{\mathrm{c}}$ as a function of the incident energy; (CERN-Dortmund-Heidelberg-Saclay experiment).]

tion significative de $\bar{B}$ ni du rapport $\bar{\sigma}_{\mathrm{c}} / \sigma_{\mathrm{c}}$ avec l'énergie. En ce qui concerne les sections efficaces, on notera que les 3 expériences précédentes dispo- sent d'une mesure du flux incident. On peut donc conclure aujourd'hui à l'absence de gros effets anormaux à haute énergie tant dans les distributions en $y$, que dans les sections efficaces inclusives.

5 Conclusions. - Nous disposons aujourd'hui d'une explication satisfaisante de toutes les données expérimentales sûres ; cette explication repose sur les points suivants :

- le modèle des quarks-partons pour la structure du nucléon;

- la structure V - A du courant faible chargé;

- le modèle de Salam-Weinberg pour la structure du courant faible neutre, avec $\sin ^{2} \theta_{\mathrm{W}} \sim 0,3$;

- l'existence d'un $4^{\mathrm{e}}$ quark intervenant dans le nouveau courant chargé introduit par Glashow, Iliopoulos et Maiani.

Les programmes expérimentaux tant à Fermi Lab. qu'au SPS du CERN visent aujourd'hui à des tests plus précis de ces modèles. En effet, si de grosses déviations par rapport à leurs prédictions semblent aujourd'hui peu vraisemblables, on ne peut exclure des effets anormaux rares, en particulier des réactions mettant en jeu de nouveaux quarks. La découverte récente d'une nouvelle particule «upsilon » [52] dans le spectre de masse $\mu^{+} \mu^{-}$et $\mathrm{e}^{+} \mathrm{e}^{-}$des paires de leptons produites dans des réactions ( $p+$ noyau) à Fermi Lab. incite à chercher dans cette direction. Enfin des surprises de plus grosse taille, tenant à la nature même du neutrino, comme l'existence d'oscillations $\nu_{\mu} \rightleftarrows \nu_{\mathrm{e}}$ ou $\nu_{\mu} \rightleftarrows \bar{\nu}_{\mu}$ suggérées par divers auteurs [53] peuvent encore bouleverser dans les prochaines années la physique du neutrino.

\section{Bibliographie}

[1] LlewellyN SMITH, C. H., in Proceedings of the Int. Symp. on Electron and Photon interactions at high energies, Bonn, 1973 (North Holland) 1974, p. 449.

[2] Hasert, F. J. et al., Phys. Lett. 46B (1973) 138.

[3] Turlay, R., Ecole d'été de Gif-sur-Yvette 1974, Tome 1, p. 205.

[4] Glashow, S. L., Iliopoulos, J., Maiani, L., Phys. Rev. D2 (1970) 1285 .

[5] AUBERT, B. et al., in Proceedings of the Seventeenth International Conference on High Energy Physics, London, 1974 (edited by J. R. Smith, Rutherford High Energy Laboratory) 1975.

[6] Blietschau, J. et al., Phys. Lett. 60B (1976) 207.

[7] SchwitTERs, R., in Proceedings of the Eighteenth International Conference on High Energy Physics, Tbilisi, 1976 (Dubna) 1977 , p. B 34.

[8] KnAPp, B. et al., Phys. Rev. Lett. 37 (1976) 882.

[9] Hung, P. Q. and Sakurai, J. J., Phys. Lett. 63B (1976) 295.

[10] SakuraI, J. J., Neutral currents without gauge theory prejudices, TH. 2099-CERN (September 1975).

[11] Blietschau, J. et al., Nucl. Phys. B114 (1976) 189. BRISSON, V., communication privée.

[12] FaIssNer, H., in Proceedings of the Eighteenth International Conference on High Energy Physics, Tbilisi, 1976 (Dubna) 1977 , p. B 114.
[13] FaIsSner, H., in Proceeding of the Neutrino 77 Conference, Elbruz, 1977 (à paraître).

[14] ReInes, F., GURR, H. S. and Sobel, H. W., Phys. Rev. Lett. 37 (1976) 315.

[15] Weinberg, S., Phys. Rev. Lett. 19 (1967) 1264.

SALAM, A., in Elementary Particle Theory; relativistic groups and analyticity, Proc. 8th Nobel Symp., Aspenägarden (1968), ed. N. Svartholm (Almquist and Wiksells, Stockholm) 1968 p. 367.

SALAM, A. and WARD, J. C., Phys. Lett. 13 (1964) 168.

[16] Voir la revue faite par Aubert dans ce congrès.

[17] De Rujula, A., Georgi, H. and Glashow, S. L., Phys. Rev. Lett. 35 (1975) 69 ; Phys. Rev. D12 (1965) 3589.

Fritzsch, H., Gell-Mann, M. and Minkowski, P., Phys. Lett. 59B (1975) 256.

Pakvasa, S., Simmons, W. A. and Tuan, S. F., Phys. Rev. Lett. 35 (1975) 702.

[18] Blietschau, J. et al., Nucl. Phys. B118 (1977) 218.

[19] Benvenuti, A. et al., Phys. Rev. Lett. 37 (1976) 1039.

[20] FISK, H. E., in Proceedings of the Eighteenth International Conference on High Energy Physics, Tbilisi, 1976 (Dubna) 1977, p. B 109.

[21] STEINBERGer, J., in Proceedings of the EPS International Conference on High Energy Physics (Budapest, 1977), à paraître. 
[22] LEE, W. et al., Phys. Rev. Lett. 37 (1976) 186.

[23] Cline, D. et al., Phys. Rev. Lett. 37 (1976) 252 ; Phys. Rev. Lett. 37 (1976) 648.

[24] Bertrand-Coremans, G. H. et al., Phys. Lett. 61B (1976) 207.

[25] Neutrino Propane Gargamelle Collaboration; Experimental study of exclusive one-pion production in all neutrinoinduced neutral current channels, à paraître.

[26] FrancoIs, T, , Etude de la production de particules étranges par des courants neutres induits par des neutrinos et des antineutrinos de 1 à $12 \mathrm{GeV}$. Thèse de $3^{e}$ Cycle, Université de Paris-Sud, juin 1977.

[27] Cabibbo, N., Phys. Rev. Lett. 10 (1963) 531.

Feynmann, R. P. and Gell-Mann, M. Phys. Rev. 109 (1958) 193.

Gell-ManN, M., Phys. Rev. 125 (1962) 1067.

[28] KhOVANSKY, V., in Proceedings of the International Neutrino Conference, Aachen, 1976, (Vieweg, Braunschweig 1977) p. 54.

[29] Benvenuti, A. et al., Phys. Rev. Lett. 35 (1975) 1199 ; Phys. Rev. Lett. 35 (1975) 1249.

[30] Barish, B. C. et al., Phys. Rev. Lett. 36 (1976) 939. MINE, P., in Comptes Rendus de la $12^{e}$ rencontre de Moriond, Flaine, 1977 (à parâtre).

[31] Holder, M. et al., Phys. Lett. 69B (1977) 377.

[32] DeDEN, H. et al., Phys. Lett. 67B (1977) 474.

[33] Bosetti, P. et al., Phys. Rev. Lett. 38 (1977) 1248.

[34] Baltay, C., in Comptes Rendus de la $12^{e}$ rencontre de Moriond, Flaine, 1977 (à paraître).

Baitay, C. et al., Phys. Rev. Lett. 39 (1977) 62.

[35] Berge, J. P. et al., Phys. Rev. Lett. 38 (1977) 266.
[36] Cazzoli, E. G. et al., Phys. Rev. Lett. 34 (1975) 1125.

[37] Berge, J. P. et al., Phys. Rev. Lett. 36 (1976) 127.

[38] Burhop, E. H. S. et al., Phys. Lett. 65B (1976) 299.

[39] DE Rujula, A., in Proceedings of the Eighteenth International Conference on High Energy Physics, Tbilisi, 1976 (Dubna) 1977, p. N 111.

[40] Benvenuti, A. et al., Phys. Rev. Lett. 38 (1977) 11 i0.

[41] Benvenuti, A. et al,, Phys. Rev. Lett. 38 (1977) 1183.

[42] GershteIN, S., in Proceedings of the Eighteenth International conference on High Energy Physicsn Tbilisi, 1976 (Dubna) 1977, p. 125.

[43] Barnett, R. M., Georgi, H. and Polttzer, H. D., Phys. Rev. Lett. 37 (1976) 1313.

[44] Benvenuti, A. et al., Phys. Rev. Lett. 36 (1976) 1478.

[45] Benvenuti, A. et al., Phys. Rev. Lett. 37 (1976) 189.

[46] Barish, B. C. et al., Phys. Rev. Lett. 35 (1975) 1316.

[47] Barish, B. C. et al., Phys. Rev. Lett. 38 (1977) 314.

[48] NeZrick, F. A., in Proceedings of the International Neutrino Conference, Aachen, 1976 (Vieweg, Braunschweig) 1977 , p. 362.

[49] BodeK, A., in Proceedings of the Neutrino 77 Conference, Elbruz, 1977 (à paraître).

[50] CUNDY, D. C., in Proceedings of the Neutrino 77 Conference, Elbruz, 1977 (à paraître).

[51] Holder, M. et al., Phys. Rev. Lett. 39 (1977) 433.

[52] Herb, S. W. et al., Phys. Rev. Lett. 39 (1977) 252.

[53] Bilenky, S. M. and Pontecorvo, B., in Proceedings of the Eighteenth International Conference on High Energy Physics, Tbilisi 1976 (Dubna) 1977, p. B 122.

Eliezer, S. and SwifT, A., Nucl. Phys. B105 (1976) 45. FrirzSCH, H. and MINKOWSKI, P., Phys. Lett. 62B (1976) 72. 